\title{
Preface
}

\section{Sleep Medicine: A Wise Investment}

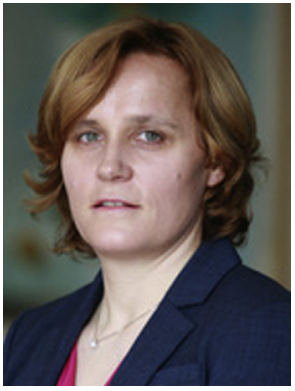

Barbara Gnidovec Stražišar, MD, PhD Editor

Sleep medicine is a young, interdisciplinary medical field that over the past decades slowly and persistently paved its way through diverse health care systems in different parts of the world. There is no doubt that good and sufficient sleep is essential for health and well-being. However, with growing demands to the health care systems brought by aging of the population and special circumstances, like the current COVID-19 pandemic, there is justified fear that diagnosing and treating sleep disorders might in the future face less affection from health care decison makers.

Conventional in-lab sleep diagnostics is often laborious and expensive; therefore, future trends in sleep medicine should be oriented to the evaluation of sleep-disordered patients in a more naturalistic home environment with monitoring devices that are less obtrusive, more accessible, and yet still reliable. Various types of ambulatory recordings have already been introduced in the clinical practice, and these should form the basis for future development of various ambulatory treatment interventions.

This special issue is addressing these challenges and offers possible solutions with new trends and evolving technology in sleep medicine. These will allow better accessibility for the detection and treatment of sleep disorders in different populations and different circumstances.
The COVID-19 pandemic reduced sleep medicine services in many parts of the world and at the same time accelerated the use of telemedicine in the field of sleep medicine, especially in the management of sleep-disordered breathing. Telemedicine is an emerging field that has currently recieved widespread acceptance among diverse medical specialities and offers also tremendous possibilities in sleep medicine for diagnosing, treatment, and long-term follow-up of sleepdisordered patients. There is, however, an obvious need for improved subjective and objective measurements of sleep. New trends in sleep medicine make use of digital management platforms for storing and presenting the data, while data science with machine learning offers new analysis of the sleep data. This can facilitate the discovery of new important patterns and novel insight in sleep data. The methodology can also help in identifying certain clinical trends for more targeted treatment and thus challenge the conventional approach to the management of sleep disorders that is often insufficient in complex phenotypes. With growing knowledge in sleep medicine, there is a parallel growth of technical solutions for lessobtrusive sensors for sleep monitoring and at the same time more data processing for improving patient management strategies. 
These are essential since sleep is important for every aspect of our health. Sleep disorders are associated with deterioration in human body functioning with increased cardiovascular risks, which can lead to negative consequences favoring the development of neurologic disorders. More prevalent sleep disorders, such as insomnia, are associated also with great economic and social burden, where direct and indirect costs far outweigh the burden of the treatment. Thus, in the future, we must be more aware also of important aspects of sleep medicine for the general public well-being, such as the importance of sleep and sleep-disordered breathing for traffic regulation and the influence of seasonal changing of the clock to human sleep.

Barbara Gnidovec Stražišar, MD, PhD General Hospital Celje College of Nursing in Celje Mariborska cesta 7 3000 Celje, Slovenia

E-mail address: barbara.gnidovec-strazisar@sb-celje.si 